\title{
Metodologia para identificar áreas prioritárias para infraestrutura verde ${ }^{1}$
}

\section{Methodology to identify priority areas for green infrastructure}

\section{Solera, Maria Lucia'; Souza, Caroline Almeida2; Machado, Aline Ribeiro3; Longo, Mariana Hortelani Carneseca4; Velasco, Giuliana Del Nero5; Amaral, Raquel Dias De Aguiar Moraes ${ }^{6}$; Ikematsu Priscila ${ }^{7}$}

1 Instituto de Pesquisas Tecnológicas, Av. Prof. Almeida Prado, 532, Brasil - lucinha @ipt.br

2 Instituto de Pesquisas Tecnológicas - caroline@ipt.br

3 Instituto de Pesquisas Tecnológicas - asribiro@ipt.br

4 Instituto de Pesquisas Tecnológicas - marihc@ipt.br

${ }^{5}$ Fundação Instituto de Pesquisas Tecnológicas - velasco@ipt.br

6 Instituto de Pesquisas Tecnológicas - raquel@ipt.br

7 Instituto de Pesquisas Tecnológicas - priscilai@ipt.br

\section{RESUMO}

As áreas urbanas apresentam cenários de degradação que poderiam ser evitados com o uso de técnicas sustentáveis que ampliam a provisão de serviços ambientais, como as que compõem a infraestrutura verde (IV) das cidades. A preservação e restauração da paisagem natural, como florestas, banhados e áreas de inundação, são componentes essenciais da IV. Quando estas áreas sensíveis são protegidas ou implantadas, há melhora na qualidade da água e nas condições do habitat da vida silvestre e geram-se oportunidades de recreação para aumento da qualidade de vida dos cidadãos. Desenvolver uma metodologia que possa identificar e priorizar áreas para implantação de determinada tipologia de IV é o objetivo do projeto de pesquisa do IPT, com apoio financeiro da Fundação IPT (FIPT). A base do projeto foi identificar as funções ambientais relacionadas às tipologias de IV e respectivos indicadores. Foram identificados nove serviços ambientais associados à IV e respectivos indicadores, detalhando-se a metodologia de obtenção dos dados e demais informações sobre as funções ambientais da IV. O trabalho contribui para o subtema Meio ambiente urbano, com a análise do campo de aplicação de 18 tipologias de IV, bem como com a apresentação dos serviços ambientais associados à IV.

Palavras-Chave: Infraestrutura Verde, Serviços Ambientais, Indicadores Ambientais

\section{ABSTRACT}

Urban areas have degradation scenarios that could be avoided by using sustainable techniques that enhance the provision of environmental services, such as those that compose the green infrastructure (GI) of cities. The preservation and restoration of the natural

\footnotetext{
${ }^{1}$ SOLERA, Maria Lucia; SOUZA, Caroline Almeida; MACHADO, Aline Ribeiro; LONGO, Mariana Hortelani Carneseca; VELASCO, Giuliana Del Nero; AMARAL, Raquel Dias de Aguiar Moraes, IKEMATSU, Priscila. Metodologia para identificar áreas prioritária para infraestrutura verde. In: II SIMPÓSIO NACIONAL DE GESTÃO E ENGENHARIA URBANA: SINGEURB, 2019, São Paulo. Anais... Porto Alegre: ANTAC, 2019.
} 
landscape, such as forests, wetlands and floodplains, are essential components of Gl. When these sensitive areas are protected or implemented, there are improvements in water quality and in the conditions of wildlife habitat and recreation opportunities are created, increasing the quality of life of citizens. Developing a methodology that can identify and prioritize areas for implementation of a specific GI typology is the objective of the IPT research project, with financial support from the IPT Foundation (FIPT). The basis of the project was to identify the environmental functions related to the typologies of IV and respective indicators. Nine environmental services associated with $G$ and related indicators were identified and the methodology for data collection and further information on the environmental functions of $\mathrm{Gl}$ were detailed. This work contributes to the sub-theme Urban environment, with the analysis of the field of application of 18 typologies of $\mathrm{Gl}$, as well as with the presentation of the environmental services associated with $\mathrm{Gl}$.

Key words: Green Infrastructure, Environmental Services, Environmental Indicators

\section{INTRODUÇÃO}

Sabendo-se que as áreas urbanas apresentam diversos cenários de degradação, com alto custo de recuperação e que poderiam ser evitados com o uso de técnicas sustentáveis, a infraestrutura verde (IV) surge como uma alternativa para aliar a mitigação destes cenários na paisagem urbana com a provisão de serviços ambientais essenciais para a sustentabilidade das cidades. Porém, para tornar essa alternativa viável, um dos desafios é definir as áreas prioritárias a implementar determinadas tipologias de IV para prover os serviços ambientais desejados. Nesse sentido foi necessário aprofundar o entendimento dos temas: serviços ambientais, tipologias de IV e sua interação para a melhoria da qualidade de vida nas cidades.

Os serviços ambientais são os benefícios à qualidade de vida, provenientes de intervenções humanas, por meio de práticas de manejo dos recursos naturais (CHOMITZ; BRENES; CONSTANTINO, 1999; KROEGER, 2013; SCHOMERS; MATZDORF; 2013; WUNDER, 2015). Quanto aos serviços ecossistêmicos, estes são os benefícios gerados às pessoas, fornecidos pelos ecossistemas naturais, conceito adotado por Costanza et al. (1997), pela Millennium Ecosystem Assessment (2003) e pela Organização das Nações Unidas para Alimentação e Agricultura (2019). Entende-se a importância dessa distinção para qualificar os benefícios advindos de intervenções humanas por meio da adoção de Soluções baseadas na Natureza (SbN), nesse caso, os serviços ambientais, diferente dos advindos de ecossistemas naturais, não relacionados às práticas de manejo, seja de restauração ou de conservação desses ecossistemas, os serviços ecossistêmicos.

A IV é entendida como sendo uma rede interconectada de áreas verdes naturais e outros espaços abertos que conservam valores e funções ecológicas, sustentam ar e água limpos e ampla variedade de benefícios para as pessoas e para a vida silvestre, devendo orientar as ações de planejamento e desenvolvimento territoriais garantindo a existência dos processos vivos no futuro. É uma estrutura ecológica para a saúde ambiental, social e econômica (our natural life-support system) (BENEDICT; MCMAHON, 2006; FRANCO, 2010 apud BONDAR; HANNES, 2014).

A IV pode ocorrer em múltiplas escalas; da escala da paisagem à escala de projeto onde incidem os elementos cruciais da estratégia de implementação, como as práticas de desenvolvimento de baixo impacto (low impact development - LID), que são componentes indispensáveis para qualquer plano bem-sucedido de IV (BENEDICT, 2010 apud MASCARÓ, 2016). A necessidade de um planejamento articulado com outras infraestruturas e outros aspectos relevantes ao planejamento urbano e a participação da comunidade, necessária na identificação de áreas problemáticas e potenciais para sua implantação, são dois aspectos considerados na IV (MASCARÓ, 2016).

Para outros autores, a IV pode promover espaços verdes multifuncionais e se integrar com a infraestrutura cinza (IC), sendo capaz de agregar múltiplos serviços ambientais. Quanto aos aspectos multifuncionais e multiescala da IV, estes são destinados a fortalecer as conexões 
entre diferentes tipos de espaços verdes, assim como a IC. Como sugerido pela Comissão Europeia, a IV pode auxiliar na promoção de cidades compactas, enquanto que a sua redução, como resultado da ocupação urbana, pode degradar os serviços ambientais (ARTMANN; BASTIAN; GRUNEWALD, 2017).

A IV integra áreas naturais aos diversos usos do solo, como habitação, agricultura e transporte, inserindo o verde e aumentando os benefícios gerados na área ambiental, que são os serviços ambientais e no social, com a criação de empregos - negócios verdes, melhoria da saúde etc. (COPELAND, 2014). A IV também pode ser vista como uma alternativa de adaptação das cidades às mudanças climáticas e, no meio urbano, consiste de uma rede multifuncional verde-azul - vegetação - sistemas hídricos/drenagens - que incorpora o retrofit e adaptação da infraestrutura existente. (MARUYAMA; LEITE; DEUS, 2017, MOURA; PELLEGRINO; MARTINS, 2014, HERZOG; ROSA, 2010; NOVOTNY; AHERN; BROWN, 2010). Para Madureira (2012), os benefícios e funções atribuídos às áreas verdes são amplos e vem se constituindo em uma premissa importante das políticas públicas e programas de desenvolvimento sustentável.

Por fim, a IV é um instrumento que permite obter benefícios ecológicos, econômicos e sociais por meio de SbN para auxiliar a compreender as vantagens oferecidas à sociedade humana e para mobilizar investimentos que sustentem e valorizem estes benefícios (EUROPEAN ENVIRONMENT AGENCY, 2017).

O presente trabalho faz parte de um projeto de pesquisa desenvolvido pelo Instituto de Pesquisas Tecnológicas do Estado de São Paulo (IPT), financiado pela Fundação de Apoio ao IPT (FIPT), que objetiva desenvolver uma metodologia para auxiliar na identificação e priorização de áreas para implantação de IV no âmbito municipal. O objetivo principal deste artigo é apresentar os resultados parciais do projeto, com ênfase à identificação das funções ambientais relacionadas às tipologias de IV e seus respectivos indicadores.

\section{MÉTODO}

Diferentes são os conceitos atribuídos à IV, dependendo do contexto em que será aplicada. A IV pode estar relacionada aos elementos vegetais, promovendo os benefícios ecológicos em áreas urbanas ou às estruturas de engenharia para manejo das águas pluviais ou para tratamento de águas, projetadas para serem ecológicas (BENEDICT; McMAHON, 2006 apud VASCONCELLOS, 2015).

Este trabalho adotou o conceito de que a IV opera primeiro na escala de paisagem, pensando na conexão da vida silvestre, que necessariamente maximiza a cobertura florestal. Nas áreas urbanas, a IV abrange, primeiramente, a cobertura das copas das árvores: as condições da arborização urbana; florestas ripárias; a conexão entre os parques da cidade; corredores verdes; e jardins comunitários. Na escala local, destacam-se as áreas com necessidade de instalação de jardins de chuva; telhados verdes; pavimentos permeáveis; e outras práticas de infiltração da água de chuva (FIREHOCK, 2010).

O trabalho mostra as três primeiras etapas de projeto de pesquisa para desenvolver uma metodologia, com vistas a auxiliar os gestores públicos a identificar e priorizar áreas para a implantação da IV no âmbito municipal, como alternativa para melhorar a qualidade de vida nas cidades dentro de programas de políticas públicas do estado de São Paulo e de desenvolvimento sustentável.

Sendo assim, o projeto teve como base identificar as funções ambientais relacionadas às tipologias de IV e seus respectivos indicadores a partir de etapas sintetizadas e apresentadas na Figura 1. 
Figura 1 - Etapas do projeto de pesquisa. Em "cinza" as etapas abordadas neste trabalho.

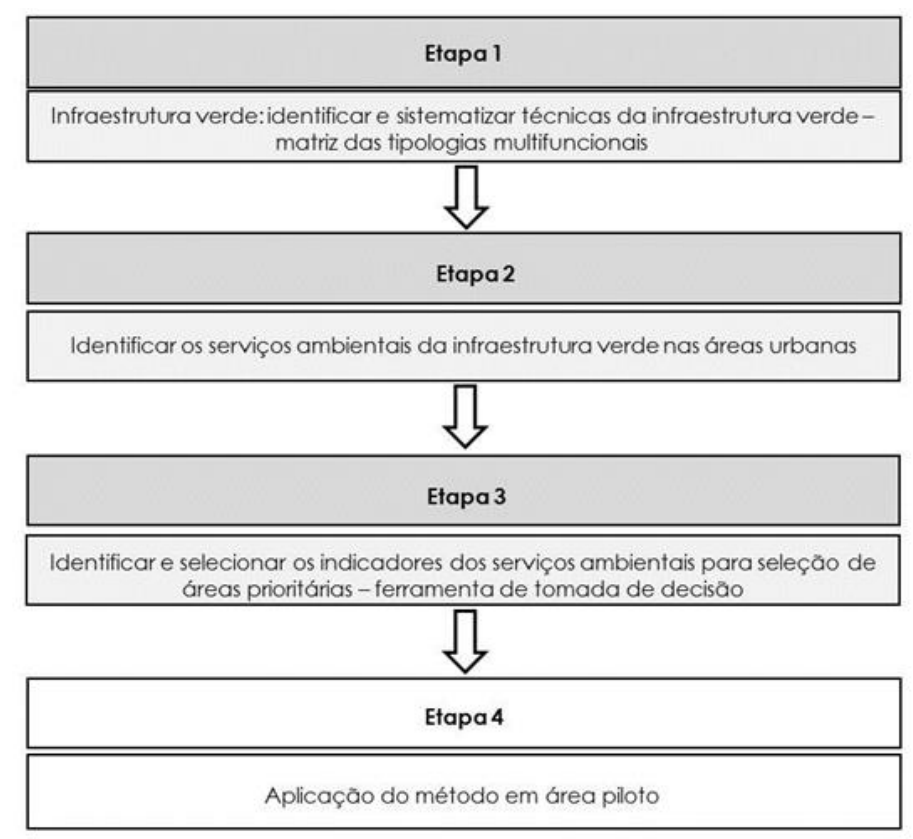

Fonte: Os autores

A Ełapa 1 consistiu da elaboração do Estado da Arte da IV com vistas ao entendimento dessa tecnologia/estratégia para identificar as tipologias multifuncionais disponíveis na literatura, as quais foram sistematizadas em uma matriz, contendo informações tais como: descrição, campo de aplicação, vantagens e limitações.

Paralelo à Ełapa 1, foram elencados os diversos serviços ambientais relacionados à IV (Ełapa 2), os quais foram agrupados nas categorias de serviços de regulação, de provisão, culturais e de suporte, conforme a classificação proposta na Avaliação Ecossistêmica do Milênio (MA, 2003). Na sequência, por meio de revisão bibliográfica acerca dos métodos utilizados para medição dos serviços ambientais selecionados, foram realizadas a identificação e a seleção dos indicadores ambientais passíveis de serem medidos (Ełapa 3), que foram descritos utilizando "Descritores dos Indicadores Ambientais".

Esses descritores, além de organizar as informações e descrever a metodologia de obtenção dos dados, contemplam informações acerca da relação das funções ambientais da IV com as diretivas do Programa Município Verde Azul (PMVA) - programa estadual que incentiva e contribui com as prefeituras paulistas na elaboração e execução de suas políticas públicas estratégicas voltadas ao desenvolvimento sustentável em São Paulo -, e com os Objetivos do Desenvolvimento Sustentável (ODS) - planejados para facilitar sua adoção por todos os países conforme suas próprias prioridades e promover uma parceria global orientadora para as decisões presentes e futuras (ONU NO BRASIL, 2019)

A base de dados dos indicadores de serviços ambientais será estruturada de modo a receber e tratar informações georreferenciadas de diferentes naturezas, compondo o sistema para identificação de áreas prioritárias a ser operado em ambiente SIG (Sistemas de Informação Geográfica). A sobreposição de dados cartográficos para se obter uma síntese da paisagem e de seus processos atuantes, consiste de um procedimento viabilizado com o emprego de tecnologias de geoprocessamento, muito utilizado ao lado de sistemas de apoio à tomada de decisões (decision support systems), para decisões de planejamento transparentes e que possam ser repetidas intersubjetivamente.

Por fim, a Ełapa 4 consistirá na aplicação da ferramenta de tomada de decisão em área piloto, com o intuito de validar e aperfeiçoar o método desenvolvido. 


\section{RESULTADOS E DISCUSSÃO}

As técnicas de IV sistematizadas a partir de revisão bibliográfica resultaram na seleção de algumas tipologias mais comumente citadas na literatura, apresentadas na Figura 1 , contendo informações úteis para a identificação dos serviços ambientais associados à IV, bem como para a priorização de áreas para implantação da IV como: como campo de aplicação e, algumas vantagens e limitações da implantação de IV.

A definição das áreas prioritárias para implantação de IV será realizada a partir da análise das regiões com maior quantidade de características favoráveis à geração e manutenção dos serviços ambientais. A Figura 2 mostra detalhadamente as funções ambientais por categoria de serviço ambiental, com seus respectivos indicadores e métricas.

Essas informações serão utilizadas para identificar as áreas prioritárias para a instalação de IV, tendo como área piloto, a subprefeitura do Butantã e seus distritos (Butantã, Raposo Tavares, Rio Pequeno, Vila Sônia e Morumbi), assim como para calibrar a metodologia e poder indicar quais das tipologias multifuncionais da IV podem ser implantadas nas áreas prioritárias identificadas com a aplicação do método. 
Figura 1 - Tipologias para compor a infraestrutura verde

\begin{tabular}{|c|c|c|c|}
\hline Tipologia & C ampo de Aplicação & Vartagens & Limitaçöes \\
\hline $\begin{array}{l}\text { J ardim de } C \text { huva } \\
\text { (LID) }\end{array}$ & $\begin{array}{l}\text { - áreasresidenciais } \\
\text { - vias unbanas próximas ao } \\
\text { meio fio } \\
\text { - hortas }\end{array}$ & $\begin{array}{l}\text { - purificação das águas pluviais } \\
\text { - manutenção da biodiversidade } \\
\text { - redução de ilhas de calor } \\
\text { - captura de } \mathrm{CO}_{2}\end{array}$ & $\begin{array}{l}\text { - permeabilidade do } \\
\text { - volo } \\
\text { variação do lençol } \\
\text { freátic }\end{array}$ \\
\hline $\begin{array}{l}\text { Canteiro Pluvial } \\
\text { (LID) }\end{array}$ & $\begin{array}{l}\text { - vias unbanas próximas ao } \\
\text { meio fio } \\
\text { - edifícios }\end{array}$ & $\begin{array}{l}\text { - purificação das águas pluviais } \\
\text { - reduça do escoamento superficial } \\
\text { - redução de ilhas de calor } \\
\text { - promoção da biodiversidade } \\
\text { - captura de } \mathrm{CO}_{2}\end{array}$ & $\begin{array}{l}\text { - risc o de contaminar } \\
\text { o so lo } \\
\text { - risc o de contaminar } \\
\text { o lenç ol freátic o }\end{array}$ \\
\hline $\begin{array}{l}\text { Biovaleta } \\
\text { (LID) }\end{array}$ & $\begin{array}{l}\text { - vias unbanas próximas ao } \\
\text { meio fio } \\
\text { - estacionamentos }\end{array}$ & $\begin{array}{l}\text { - redução do escoamento superficial } \\
\text { - reposiça ão do lenç ol freático } \\
\text { - elemento estético }\end{array}$ & $\begin{array}{l}\text { - não recomendada } \\
\text { para áreas } \\
\text { densamente } \\
\text { urbanizadas } \\
\end{array}$ \\
\hline $\begin{array}{l}\text { Pavimento } \\
\text { Drenante } \\
\text { (LID) }\end{array}$ & $\begin{array}{l}\text { - calçadas } \\
\text { - estacionamentos } \\
\text { - quintais residenciais } \\
\text { - espaços públic os de lazer }\end{array}$ & $\begin{array}{l}\text { - redução do escoamento superficial } \\
\text { - recarga do lençol freático } \\
\text { - filtragem de alguns poluentes } \\
\text { - redução de ac úmulo de água da } \\
\text { chuva }\end{array}$ & $\begin{array}{l}\text { - risc o de contaminar } \\
\text { o lençol freátic o }\end{array}$ \\
\hline $\begin{array}{l}\text { Lagoa Seca/ } \\
\text { Bacia de } \\
\text { Detenção } \\
\text { (LID) }\end{array}$ & $\begin{array}{l}\text { - vias urbanas } \\
\text { - ros } \\
\text { - parques lineares } \\
\text { - jardins públicose privados }\end{array}$ & - redução do escoamento superficial & - näo identific ada \\
\hline $\begin{array}{l}\text { Lagoas Pluviais } \\
\text { (LID) }\end{array}$ & - grandesáreasabertas & $\begin{array}{l}\text { - amazenamento de grandes } \\
\text { quantidades de água } \\
\text { - recuperação da qualidade da água } \\
\text { - cooperação com o habitat }\end{array}$ & $\begin{array}{l}\text { - nec esaidade de } \\
\text { grandesáreas }\end{array}$ \\
\hline $\begin{array}{l}\text { Cistemas } \\
\text { (LID) }\end{array}$ & $\begin{array}{l}\text { - áreasque desejam } \\
\text { aumentara eficiência do } \\
\text { uso dasáquasdasc huvas }\end{array}$ & & - não identific ada \\
\hline $\begin{array}{l}\text { A.lagados } \\
\text { C anstruídos } \\
\text { (LID) }\end{array}$ & - ambientes urbanos & $\begin{array}{l}\text { - purificação das águas pluviais } \\
\text { - promoção da retençăo/remoção de } \\
\text { contaminantes }\end{array}$ & - não identific ada \\
\hline $\begin{array}{l}\text { Bioengenharia de } \\
\text { Solos } \\
\text { (LID) }\end{array}$ & $\begin{array}{l}\text { - taludes, encostase margens } \\
\text { fluviais degradadas }\end{array}$ & $\begin{array}{l}\text { - aumento da estabilidade de enc ostas } \\
\text { - melhoria do regime hídrico do solo } \\
\text { - criação e provisão de habitats }\end{array}$ & $\begin{array}{l}\text { - mão de obra } \\
\text { especializada } \\
\text { - limitação no perído } \\
\text { de domência das } \\
\text { sementes } \\
\text { - disponibilidade de } \\
\text { espécies adaptadas } \\
\text { àscondições locais }\end{array}$ \\
\hline Agricultura Urbana & $\begin{array}{l}\text { - espaçosresiduais } \\
\text { - áreasnão oc upadas } \\
\text { - fachadas }\end{array}$ & - socialização e educ ação & - não identific ada \\
\hline Ruasverdes & - arborzação de nas & $\begin{array}{l}\text { - conexão para avifauna e microfauna } \\
\text { - amenização do clima } \\
\text { - lim ita o trafego de veíc ulo spesados } \\
\text { - redução da carga difusa }\end{array}$ & - não identific ada \\
\hline $\begin{array}{l}\text { Viasde Usos } \\
\text { Múltiplos }\end{array}$ & - nas arborzadas & $\begin{array}{l}\text { - concilia diversos usos como veículos, } \\
\text { pedestrese ciclovias seguras }\end{array}$ & - não identific ada \\
\hline Interseções Viárias & - vias urbanas & $\begin{array}{l}\text { - organização viária } \\
\text { - coleta de água das chuvas } \\
\text { - plantio de espécies nativas } \\
\text { - criação e provisão de habitat } \\
\text { - amenização do mic roc lima } \\
\text { - melhoria do visual estétic o }\end{array}$ & - não identific ada \\
\hline Telha do Verde & $\begin{array}{l}\text { - recobrimento da cobertura } \\
\text { de edificaçöes }\end{array}$ & $\begin{array}{l}\text { - detenção e retardamento da } \\
\text { entradas daságuasno sistema de } \\
\text { drenagem } \\
\text { - redução da temperatura intema das } \\
\text { edificações } \\
\text { - criação e provisão de habitat }\end{array}$ & $\begin{array}{l}\text { - ocorrência de } \\
\text { infiltração e umidade } \\
\text { na edificação }\end{array}$ \\
\hline $\mathrm{J}$ ardins $v$ ertic a is & $\begin{array}{l}\text { - qualquer superfície que } \\
\text { esteja delim itada } \\
\text { verticalmente }\end{array}$ & - melhoria do conforto témico & $\begin{array}{l}\text { - problemascom } \\
\text { umidade }\end{array}$ \\
\hline Grade verde & $\begin{array}{l}\text { - áreascom solos argilosose } \\
\text { topografia íngreme }\end{array}$ & - melhoria do fluxo de drenagem & - não identific ada \\
\hline $\begin{array}{c}\text { Corre dores Verdes } \\
\text { Unbanos }\end{array}$ & $\begin{array}{l}\text { - espaçoslivres para } \\
\text { recreação }\end{array}$ & $\begin{array}{l}\text { - conexão dosfragmentos de } \\
\text { vegetação } \\
\text { - melhoria do mic roclima } \\
\text { - manutenção da biodiversidade } \\
\text { - proteção dosc ursos d'água } \\
\end{array}$ & - não identific ada \\
\hline $\begin{array}{l}\text { Areasverdes } \\
\text { Unbanas }\end{array}$ & $\begin{array}{l}\text { - unidades de c onsevação } \\
\text { - áreasde preservação } \\
\text { permanente } \\
\text { - parques munic ipais } \\
\text { - áreaspúblicas não } \\
\text { edificadas }\end{array}$ & $\begin{array}{l}\text { - melhoria do mic roclima } \\
\text { - melhoria da qualidade do ar } \\
\text { - proteção do so lo e comosd 'água } \\
\text { - refenuaça ão do de sequilibrio climático } \\
\text { - qualidade de vida sivestre }\end{array}$ & - não identific ada \\
\hline
\end{tabular}

Fonte: Os autores com dados de Barbosa e Castro Fonte (2016); Cormier e Pellegrino (2008); Herzog e Rosa (2010); Mascaró (2016) 
Figura 2 - Indicadores e serviços ambientais relacionados a IV

\begin{tabular}{|c|c|c|c|}
\hline Função Ambiental & \multicolumn{2}{|c|}{ Serviç o Ambiental da IV } & $\begin{array}{l}\text { Indic adores Ambienta is } \\
\text { (métricas) }\end{array}$ \\
\hline \multirow{6}{*}{$\begin{array}{l}\text { Proteção dos Recursos } \\
\text { Híd ricose Solo }\end{array}$} & \multirow{6}{*}{ Regulação } & $\begin{array}{l}\text { manutenção da } \\
\text { va zã o híd ric a }\end{array}$ & $\begin{array}{c}\text { área impermeável em relação à } \\
\text { área da bacia de contribuição } \\
\text { de cada na scente (\%) }\end{array}$ \\
\hline & & \multirow{3}{*}{$\begin{array}{l}\text { melhoria da } \\
\text { qualidade da água }\end{array}$} & $\begin{array}{c}\text { índice de potencial para } \\
\text { geração de sedimentos } \\
\text { (a dimensional - qualita tivo) }\end{array}$ \\
\hline & & & $\begin{array}{c}\text { área desprotegida dos cursos } \\
\text { d'água }\left(\mathrm{km}^{2}\right)\end{array}$ \\
\hline & & & $\begin{array}{c}\text { tota I de á reas impermeá veis nas } \\
\text { bac ias hid rog rá fic as }(\%)\end{array}$ \\
\hline & & \multirow[t]{2}{*}{$\begin{array}{l}\text { mitig ação eventos } \\
\text { híd ric os extremos }\end{array}$} & $\begin{array}{c}\text { densid ade de ocorrencias de } \\
\text { a la gamentos registra dos em } \\
\text { óna os de defesa contra os } \\
\text { desastres natura is ( } \mathrm{N}^{\circ} \mathrm{de} \\
\left.\text { pontos } \mathrm{km}^{2}\right)\end{array}$ \\
\hline & & & $\begin{array}{c}\text { á reascom relevo plano no } \\
\text { entomo de rios c analizados } \\
\left(\mathrm{km}^{2}\right)\end{array}$ \\
\hline \multirow{2}{*}{$\begin{array}{c}\text { Biodiversid ade e Fluxo } \\
\text { Gênico de Fa una e } \\
\text { Flora }\end{array}$} & \multirow[b]{2}{*}{ Suporte } & $\begin{array}{c}\text { manutenção de } \\
\text { habitat }\end{array}$ & $\begin{array}{c}\text { área com cobertura vegetal } \\
\text { nativa existente em rela çã o à } \\
\text { á rea total }\left(\mathrm{km}^{2}\right)\end{array}$ \\
\hline & & $\begin{array}{l}\text { manutenção da } \\
\text { diversidade } \\
\text { genétic a (fluxo } \\
\text { gênic o) }\end{array}$ & $\begin{array}{c}\text { índ ice de área potencial para } \\
\text { criação de corred ores } \\
\left.\text { ecológ ic os (área } \mathbf{s}^{2} \mathrm{~m}^{2}\right)\end{array}$ \\
\hline \multirow{2}{*}{$\begin{array}{c}\text { Bem estardas } \\
\text { Populações Huma nas }\end{array}$} & \multirow{2}{*}{ Cultural } & $\begin{array}{l}\text { recreação / sa úde } \\
\text { físic a e mental }\end{array}$ & $\begin{array}{c}\mathrm{n}^{\circ} \text { de habita ntes que residem } \\
\text { perto das áreas verdes ( } \mathrm{n}^{\circ} \mathrm{de} \\
\text { habitantes) }\end{array}$ \\
\hline & & $\begin{array}{c}\text { diminuir a } \\
\text { vulnera bilid ade } \\
\text { social }\end{array}$ & $\begin{array}{l}\text { índice de vulnerabilid ade social } \\
\text { (índ ice admensional) }\end{array}$ \\
\hline \multirow[b]{2}{*}{ Equilíbrio Ambiental } & \multirow[b]{2}{*}{ Regulação } & $\begin{array}{l}\text { redução do efeito } \\
\text { de ilhas de calor }\end{array}$ & temperatura de superfíc ie $\left({ }^{\circ} \mathrm{C}\right)$ \\
\hline & & $\begin{array}{l}\text { melhoria da } \\
\text { qualidade do ar }\end{array}$ & $\begin{array}{c}\text { densid ade de ánores nas ruas } \\
\text { com gra nde trânsito de veíc ulos } \\
\left(\mathrm{n}^{\circ} \mathrm{de} \text { ánores } / \mathrm{m}^{2}\right)\end{array}$ \\
\hline
\end{tabular}

Fonte: Os autores

\section{CONCLUSÃO}

Este trabalho mostra os resultados obtidos nas três primeiras etapas do projeto de pesquisa desenvolvido pelo IPT relacionadas à IV, identificação dos serviços ambientais e indicação e seleção dos indicadores ambientai

Com a metodologia desenvolvida, espera-se que os gestores públicos sejam capazes de identificar as áreas em seus municípios com os maiores déficits de funções ambientais relacionadas à proteção dos recursos hídricos e solo; à biodiversidade e fluxo gênico de fauna e flora; ao bem estar das populações humanas; e ao equilibrio ambiental, e de implantar as melhores soluções para a área urbana ampliando a provisão de serviços ambientais para a população.

\section{AGRADECIMENTOS}

Os autores agradecem o Instituto de Pesquisas Tecnológicas do Estado de São Paulo (IPT), pelo apoio técnico e à Fundação IPT (FIPT) pelo apoio financeiro. 


\section{REFERÊNCIAS}

ARTMANN, Martina; BASTIAN, Olaf; GRUNEWALD, Karsten. Using the concepts of green infrastructure and ecosystem services to specify Leitbilder for compact and green cities-the example of the landscape plan of Dresden (Germany). Sustainability, v. 9, n. 2, p. 198, 2017.

BARBOSA, Murilo Cruciol; CASTRO FONTES, Maria Solange Gurgel. Jardins verticais: modelos e técnicas. PARC Pesquisa em Arquitetura e Construção, v. 7, n. 2, p. 114-124, 2016.

BONDAR, Camila Simhon; HANNES, Evy. Infraestrutura verde para o bairro do Mandaqui: possibilidade ou utopia?. Revista LABVERDE, n. 9, p. 29-52, 2014.

CORMIER, Nathaniel S.; PELLEGRINO, Paulo Renato Mesquita. Infra-estrutura verde: uma estratégia paisagística para a água urbana. Paisagem e Ambiente, n. 25, p. 127-142, 2008.

CHOMITZ, Kenneth M.; BRENES, Esteban; CONSTANTINO, Luis. Financing environmental services: the Costa Rican experience and its implications. Science of the Total Environment, v. 240, n. 1-3, p. 157-169, 1999.

COPELAND, Claudia. Green Infrastructure and issues in managing urban stormwater. Washington, DC: Library of Congress, Congressional Research Service, 2014.

COSTANZA, Robert et al. The value of the world's ecosystem services and natural capital. Nature, v. 387, p. 253-260, 1997.

EUROPEAN ENVIRONMENT AGENCY. What is green infrastructure? Copenhagen: EEA, 2007. Disponível em: <https://www.eea.europa.eu/themes/sustainability-transitions/urbanenvironment/urban-green-infrastructure/what-is-green-infrastructure>. Acesso em: 09 abr. 2019.

FIREHOCK, Karen. A short history of the term green infrastructure and selected literature. Charlottesville: Green Infrastructure Center, 2010.

HERZOG, Cecilia Polacow; ROSA, Lourdes Zunino. Infraestrutura verde: sustentabilidade e resiliência para a paisagem urbana. Revista Labverde, n 1, p. 91-115, 2010.

KROEGER, Timm. The quest for the "optimal" payment for environmental services program: Ambition meets reality, with useful lessons. Forest Policy and Economics, v. 37, p. 65-74, 2013.

MARUYAMA, Cintia Miua; LEITE, Laís Padilha; DE DEUS, Lívia Borges Dualibe. Corredor de infraestrutura verde: rota cicloviária como conexão entre Parque do Povo-lbirapuera.

Revista LABVERDE, v. 8, n. 1, p. 65-90, 2017.

MASCARÓ, Juan Luis (Org.). Infraestrutura Verde para o século XXI. Porto Alegre: Masquatro Editora, 2016.

MADUEIRA, Helena. Infra-estrutura verde na paisagem urbana contemporânea: o desafio da conectividade e a oportunidade da multifuncionalidade. Geografia: Revista da Faculdade de Letras da Universidade do Porto, n. 1, 2012.

MILLENNIUM ECOSYSTEM ASSESSMENT. Ecosystems and human well-being: a framework for assessment. Washington, DC: Island Press, 2003. 245 p.

MOURA, Newton Celio Becker; PELLEGRINO, Paulo Renato Mesquita; MARTINS, José Rodolfo Scarati. Transição em infraestruturas urbanas de controle pluvial: uma estratégia paisagística de adaptação às mudanças climáticas. Paisagem e Ambiente, n. 34, p. 107-128, 2014. 
NOVOTNY, Vladimir; AHERN, Jack; BROWN, Paul. Water Centric Sustainable Communities: planning, retrofitting, and building the next urban environment. New York: John Wiley \& Sons, 2010.

ONU NO BRASIL. A Agenda 2030 para o Desenvolvimento Sustentável. 2018. Disponível em: <http://www.agenda2030.com.br/sobre/>. Acesso em: 4 jan. 2019.

ORGANIZAÇÃO DAS NAÇÕES UNIDAS PARA ALIMENTAÇÃO E AGRICULTURA. Ecosystem services and biodiversity (ESB). Disponível em: <http://www.fao. org/ecosystem-servicesbiodiversity/en/>. Acesso em: 08 abr. 2019.

SCHOMERS, Sarah; MATZDORF, Bettina. Payments for ecosystem services: A review and comparison of developing and industrialized countries. Ecosystem services, v. 6, p. 16-30, 2013.

VASCONCELLOS, Andréa Araujo. Infraestrutura verde aplicada ao planejamento da ocupação urbana. Curitiba: Appris Editora e Livraria Eireli-ME, 2015.

WUNDER, Sven. Revisiting the concept of payments for environmental services. Ecological Economics, v. 117, p. 234-243, 2015. 